Published in final edited form as:

Int J Cardiol. 2017 March 01; 230: 420-426. doi:10.1016/j.ijcard.2016.12.091.

\title{
Depression and chest pain in patients with coronary artery disease
}

\author{
Salim S. Hayek ${ }^{a, 1}$, Yi-An Ko a,b,1, Mosaab Awad ${ }^{a, 1}$, Andrea Del Mar Soto ${ }^{a, 1}$, Hina Ahmeda, , $^{a}$ \\ Keyur Patel $^{\mathrm{a}, 1}$, Michael Yuan ${ }^{\mathrm{c}, 1}$, Spencer Maddox ${ }^{\mathrm{d}, 1}$, Brandon Gray ${ }^{\mathrm{a}, 1}$, Jamal Hajjari ${ }^{\mathrm{d}, 1}$, \\ Laurence Sperling $^{\mathrm{a}, 1}$, Amit Shah ${ }^{\mathrm{a}, \mathrm{c}, 1}$, Viola Vaccarino ${ }^{\mathrm{a}, \mathrm{c}, 1}$, and Arshed A. Quyyumi ${ }^{\mathrm{a},{ }^{*}, 1}$ \\ aDivision of Cardiology, Emory University School of Medicine, Atlanta, GA, United States \\ ${ }^{b}$ Department of Biostatistics and Bioinformatics, Rollins School of Public Health, Emory \\ University, Atlanta, GA, United States \\ 'Department of Epidemiology, Rollins School of Public Health, Emory University, Atlanta, GA, \\ United States \\ ${ }^{\mathrm{d} D e p a r t m e n t}$ of Medicine, Emory University School of Medicine, Atlanta, GA, United States
}

\begin{abstract}
Background-Depression is common in patients with coronary artery disease (CAD) and is associated with more frequent chest pain. It is however unclear whether this is due to differences in underlying CAD severity. We sought to determine [1] whether depressive symptoms are associated with chest pain independently of CAD severity, [2] whether improvement in depressive symptoms over time is associated with improvement in chest pain and [3] whether the impact of revascularization on chest pain differs between patients with and without depression.
\end{abstract}

Methods and results -5158 patients (mean age $63 \pm 12$ years, $65 \%$ male, $20 \%$ African American) undergoing cardiac catheterization completed the Seattle Angina Questionnaire (SAQ) and Patient Health Questionnaire-8 (PHQ-8) to assess angina severity and screen for depression, respectively, both at baseline and between 6 and 24 months of follow-up. We found significant correlations between PHQ-8 scores and angina frequency (SAQ-AF, $r=-0.28$ ), physical limitation (SAQ-PL, $r=-0.32$ ) and disease perception (SAQ-DS $r=-0.37$, all $P<0.001$ ), which remained significant after adjustment for clinical characteristics, CAD severity, and antidepressant use. Improvement in depressive symptoms at follow-up was associated with improvement in angina subscales (SAQ-AF $\beta$ 1.34, $P<0.001$ ), SAQ-PL $\beta$ 1.85, $P<0.001$ ), and SAQ-DS $(\beta 2.12, P<$ 0.001 ), independently of CAD severity or revascularization. Patients with depression who underwent revascularization had less improvement in chest pain frequency than those without depressive symptoms.

\footnotetext{
"Corresponding author at: Division of Cardiology, Department of Medicine, Emory University School of Medicine, 1462 Clifton Rd. NE, Suite 507, Atlanta, GA 30322, United States. aquyyum@emory.edu (A.A. Quyyumi).

${ }^{1}$ All authors take responsibility for all aspects of the reliability and freedom from bias of the data presented and their discussed interpretation.

Supplementary data to this article can be found online at http://dx.doi.org/10.1016/j.ijcard.2016.12.091.

Conflict of interest

None of the authors have conflicts of interest to disclose.
} 
Conclusions-Depression is associated with angina, independently of CAD severity. Patients with depression may not derive as adequate symptomatic benefit from revascularization as those without. Whether treatment of underlying depression improves chest pain needs to be further studied.

\section{Keywords}

PHQ-8; Angina; SAQ; Seattle Angina Questionnaire; Revascularization; Depression

\section{Introduction}

Angina pectoris or chest pain attributed to myocardial ischemia is the hallmark of obstructive coronary artery disease (CAD) and occurs as the initial manifestation of CAD in over $50 \%$ of patients with the disease [1,2]. Chest pain is a common presentation in both outpatient and inpatient settings and is often suspected to be cardiac in origin in patients with cardiovascular risk factors, even when atypical [3-5]. It however has a broad differential diagnosis involving many systems including gastrointestinal, musculoskeletal and psychiatric, and is thus neither sensitive nor specific for obstructive CAD [3-5].

Chest pain and depression commonly co-exist [6,7]. Over 30\% of patients with CAD suffer from depressive symptoms, a rate three-fold higher than in the general population $[8,9]$. Depression is considered to be a risk factor for CAD and is associated with poor outcomes $[2,10,11]$. Whether the association between depression and chest pain is dependent on the presence or severity of CAD, or whether this comorbidity is due to other factors, remains unclear. Both pain and depression share common neurochemical pathways [12], and a few studies have suggested that patients with non-obstructive $\mathrm{CAD}$ and depression have greater incidence of persistent chest pain $[7,13]$. Understanding the relation between depression and chest pain is important in order to provide the impetus to study whether treatment of depression improves chest pain, and whether individuals presenting with chest pain should be screened for depression.

In patients with suspected or confirmed CAD undergoing coronary angiography, we sought to determine [1] whether depressive symptoms are associated with chest pain independent of CAD severity, [2] whether improvement in depressive symptoms over time is associated with improvement in chest pain, and [3]whether the impact of revascularization on chest pain differs between patients with and without depression.

\section{Methods}

\subsection{Study design}

Patients ( $n=5158$ ) were recruited from the Emory Cardiovascular Biobank, a prospective registry of subjects undergoing cardiac catheterization for symptoms of chest pain or abnormal stress test at three Emory Healthcare sites in Atlanta, GA between 2003 and 2014 [14]. Subjects with acute myocardial infarction, congenital heart disease, severe valvular heart disease, severe anemia, recent blood transfusion, myocarditis, history of active inflammatory disease or cancer were excluded. Subjects aged 20 to 90 years were 
interviewed pre-catheterization to collect information on demographic characteristics, medical history, medication use, and behavioral habits. Medical records were reviewed to confirm self-reported medical history. Self-administered Patient Health Questionnaire-8 (PHQ-8) and Seattle Angina Questionnaire (SAQ) were completed both at enrollment and between 6 and 24 months of follow-up (median of 15 months). Obstructive CAD was defined as the presence of a $\geq 50 \%$ diameter stenosis in any of the major coronary arteries by visual assessment on the angiogram as reported by the proceduralists, all of whom were blinded to the questionnaire data. Coronary artery disease severity was quantified on a continuous scale using the Gensini score [15,16]. A total of 645 (13\%) patients underwent revascularization at or post-enrollment and answered follow-up PHQ-8 questionnaires. The study complies with the Declaration of Helsinki, and was approved by the Institutional Review Board. All patients provided written informed consent.

\subsection{Assessment of depressive symptoms: the patient health questionnaire-8}

The PHQ-8 is a validated self-administered 8-item questionnaire that is used to screen for depressive symptoms and diagnose major depression, in which question 9 of the PHQ-9 questionnaire concerning suicidality is omitted (Fig. S1) [17-19]. The 8 items estimate the occurrence of depressive symptoms in the past 2 weeks, and are scored from 0 ("not at all") to 3 ("nearly every day") for a maximal score of 24 . Higher scores suggest increased severity of depression, with scores of 5, 10, 15, and 20 representing cut-points for mild, moderate, moderately severe and severe depression, respectively [17-19]. Enrollment PHQ-8 scores were available for all 5158 (100\%) patients, while follow-up PHQ-8 scores were available for 2068 (40\%) patients. To further clarify the relationship, we examined the associations between the SAQ scores and the cognitive subscale of the PHQ summing the items that relate to cognition and mood alone (questions 1, 2, 6, 7 and 8 with a maximum score of 15) [4].

\subsection{Assessment of chest pain: the Seattle Angina Questionnaire}

The SAQ is a self-administered questionnaire of 19 questions specific for patients with CAD that quantifies the frequency of chest pain (SAQ-AF), the physical limitations attributed to it (SAQ-PL), and the perception of the extent to which their disease has affected quality of life (SAQ-DP) (Fig. S2) [20]. Each score ranges from 0 to 100, with lower scores indicating more frequent pain, significant physical limitations or worse quality of life. Adopted severity cut-offs for all three scores are 75-100 for minimal, 50-74 for mild, 25-49 for moderate, and $0-24$ for severe. The SAQ has been shown to be valid, reproducible and sensitive to clinical change in this patient population [21]. Enrollment SAQ-AF, SAQ-PL and SAQ-DP scores were available for 5132 (99\%), 4212 (82\%) and 4395 (85\%) of patients, respectively, while follow-up scores were available for $585(11 \%), 271(5 \%)$ and $315(6 \%)$, respectively. Missing scores were due to [1] incomplete questionnaires and [2] changes in the study protocol leading to shortening of the questionnaires to include only SAQ-AF-related questions after 2012.

\subsection{Assessment of coronary artery disease severity: Gensini score}

The Gensini score is the most commonly used angiographic scoring system [15,16]. It correlates well with other angiographic scoring systems and ultrasound plaque burden [15]. 
The score takes into consideration the severity of lesions by angiography, the cumulative effects of multiple obstructions, and the significance of jeopardized myocardium. A score is assigned to each lesion based on the severity of stenosis as indicated by the reduction of lumen diameter. A multiplier is applied to each lesion score based upon its location in the coronary tree depending on the functional significance of the area supplied by that segment. The final Gensini score is the sum of the lesion scores.

\subsection{Statistical analysis}

Continuous variables are presented as means (standard deviation) or as medians (interquartile range), and categorical variables as proportions (\%). Histograms and ShapiroWilk test were used to determine whether variables were normally distributed. Independent sample $t$-tests and chi-square tests were used to compare continuous and categorical variables, respectively. The Spearman rank-order correlation was used to measure the strength and direction of the association between PHQ-8 and SAQ subscales, both of which had a non-normal distribution. Multivariable analyses were performed using linear regression modeling of SAQ subscales at [1] baseline, [2] follow-up and [3] for the change in SAQ subscales at follow-up, incorporating in the models the characteristics that differed significantly between those with at least mild depressive symptoms (PHQ-8 25 ) and those with minimal depressive symptoms PHQ- $8<5$. These covariates included age, gender, race, BMI, hypertension, diabetes mellitus, smoking status, Gensini score and anti-depressant therapy in addition to PHQ-8 score and baseline SAQ subscales. Interactions with gender, race, use of antidepressants and the presence of obstructive CAD were examined and subgroups analyzed if $P<0.1$ for the interaction. Relative importance analysis was performed to characterize the contribution of each variable in the models predicting SAQ subscales [22]. Predictor importance was computed by taking the utility range (via conjoint analysis) for each factor separately and dividing by the sum of the utility ranges for all factors [23]. Importance weights were rescaled to sum up to 100, and represent the percentage of variance explained in the criterion that can be attributed to predictors, and may be considered as a measure of relative effect size [22]. All analyses were performed using SAS 9.3 (Cary, NC, USA).

\section{Results}

\subsection{Study population}

Of the 5158 patients, $64 \%$ ( $n=3083$ ) had obstructive CAD at enrollment (Table 1). Over $37 \%(n=1902)$ exhibited at least mild depressive symptoms (PHQ-8 25$)$; of these, $22 \%$ ( $n=$ 1157) had mild symptoms (PHQ-8 5-9), 9\% ( $n=468$ ) had moderate symptoms (PHQ-8 from 10 to 14$), 4 \%(n=204)$ had moderately severe symptoms (PHQ-8 15-19), and only $1 \%(n=73)$ had severe symptoms. Subjects with at least mild depressive symptoms were more likely to be women ( $43 \%$ versus $30 \%, P<0.001$ ), and were more likely to be receiving therapy for depression (29\% versus $10 \%, P<0.001)$ compared to those with PHQ-8 $<5$. Prevalence of obstructive CAD and Gensini scores were similar in both groups (Table 1). Younger age $(\beta-0.40, P<0.001)$, female gender $(\beta 1.7, P<0.001)$, white race $(\beta 0.66, P=$ $0.001)$, higher body mass index $(\beta 0.029, P=0.015)$, history of smoking $(\beta 0.54, P=$ $0.001)$, hypertension $(\beta 0.78, P<0.001)$ and obstructive $\operatorname{CAD}(\beta 0.48, P=0.004)$ were 
independently associated with PHQ-8 scores. Between 21 and $44 \%$ of patients had at least mild impairment in one or more of the chest pain-related SAQ subscales. All three subscales SAQ-AF, SAQDS and SAQ-PL were strongly correlated with each other ( $\mathrm{r}$ range $0.68-$ $0.79, P<0.001$, Table S1).

\subsection{Association between depressive symptoms and chest pain}

The PHQ-8 score was significantly correlated with all three subscales of the SAQ; thus, patients with worse depressive symptoms reported increased frequency of angina $(r=-0.28$, $P<0.001)$, more severe angina-related physical limitations $(r=-0.32, P<0.001)$ and perceived their disease as severe $(r=-0.37, P<0.001)$ (Fig. 1). In multivariable analyses adjusting for age, gender, race, BMI, hypertension, diabetes mellitus, hyperlipidemia, smoking status, previous myocardial infarction, CAD severity (Gensini score) and antidepressant therapy, depressive symptoms as measured by the PHQ-8 score remained an independent predictor of each SAQ subscale (Table 2). Other variables that were independently associated with SAQ subscales were CAD severity, age, gender, and BMI (Table 2). The PHQ-8 score was the most important predictor, contributing to over $70 \%$ of the variance in the models predicting SAQ-AF, SAQ-PL and SAQ-DP (Fig. 2). The cognitive subscale (PHQcog) significantly correlated with all three SAQ subscales (SAQ-AF, $r=$ -0.25 ; SAQ-PL, $r=-0.27$, SAQ-DS $r=-0.35, P<0.0001$ ).

\subsection{Association between CAD severity, depressive symptoms, and chest pain}

We then examined whether the association between depressive symptoms and chest pain was modified by the severity of CAD (Fig. 3). In multivariable analyses adjusting for the aforementioned variables, the association between PHQ-8 and SAQ subscales was independent of obstructive CAD (Table 2). Moreover, when considering non-somatic symptoms of the PHQ questionnaire, the association between the PHQ cognitive and SAQ subscales remained similar and conclusions remained the same (Table S2).

In sensitivity analyses we found no significant interaction between depressive symptoms and gender (Table 2). There was however an interaction between antidepressant use and PHQ-8 for each of the SAQ subscales: the association between depressive symptoms and chest pain frequency was significantly weaker in patient who were receiving therapy for depression ( $\beta$ -0.88 compared to $\beta-1.45$ for those not on therapy, $P=0.002$ ). The findings were similar for both the physical limitation and disease perception scales (Table 2).We also found an interaction between depressive symptoms and the presence of obstructive CAD for both the chest pain frequency $(P=0.08)$ and physical limitation scores $(P=0.008)$, denoting a stronger association between PHQ-8 and SAQ-AF, SAQ-PL in patients with obstructive CAD compared to those without (Table 2).

\subsection{Depressive symptoms and chest pain at follow-up}

The significant correlations between the PHQ-8 scores and the SAQ-subscales were validated in patients who completed questionnaires within 6-24 months of follow-up (median of 15 months); SAQ-AF ( $r=-0.48, n=585)$, SAQ—PL $(r=-0.41, n=271)$ and SAQ-DP $(r=-0.51, n=315$, all $P<0.001)$ providing validation for our baseline findings. 
We then examined whether a change in depressive symptoms at follow-up was associated with a change in SAQ subscales after accounting for clinical characteristics, antidepressant therapy at follow-up, and whether patients had undergone revascularization in the interim. In multivariable analysis, improvement in depressive symptoms (decrease in PHQ-8) was associated with significant improvements in angina subscales (SAQ-AF $\beta-1.34, P<0.001$ ), SAQ-PL $\beta-1.85, P<0.001$, and SAQ-DS $\beta-2.12, P<0.001)$. These associations were independent of CAD severity, and whether patients were on antidepressant therapy or underwent revascularization (Table 3).

\subsection{Depressive symptoms, chest pain and revascularization}

We then investigated whether depressive symptoms were contributing to increased angina and disability or whether worse SAQ scores lead to depressive symptoms in patients with significant CAD who underwent revascularization at enrollment or prior to follow-up ( $n=$ 645). Overall, in subsets who completed the SAQ questionnaire at follow-up, chest pain frequency and disease perception were improved after revascularization (SAQ-AF mean change $13 \pm 32, P<0.001$, SAQ-DS mean change $9 \pm 31, P=0.018$ ). However, there was a slight worsening of physical limitation score (SAQ-PL mean change $-8 \pm 35, P=0.023$ ). The change in SAQ scores post-revascularization were negatively correlated with the change in PHQ-8, even after multivariable adjustment ( $\triangle \mathrm{SAQ}-\mathrm{AF} \beta=-0.30, P=0.003, \Delta \mathrm{SAQ}-\mathrm{PL}$ $\beta=-0.042, P=0.008$, and $\Delta$ SAQ-DS $\beta=-0.074, P<0.001)$.

Patients with at least mild depression at baseline $(n=350)$ had a significant decrease in depressive symptoms after revascularization (mean PHQ- 8 of $10 \pm 4$ at baseline to $5 \pm 4$ at follow-up, $P<0.001$ ), which remained significant after adjustment for age, gender and race. These findings suggest that relief of ischemia and chest pain also relieves depressive symptoms.

Moreover, we found that in patients undergoing revascularization, those without depressive symptoms had greater angina relief (increase in SAQ-AF) compared to those with at least mild depressive symptoms ( $14 \pm 32$ vs. $11 \pm 32$, respectively, $P=0.005$, Fig. 4A). There were no significant differences in the change of SAQ-PL $(P=0.23)$ and SAQ-DS $(P=0.12)$ after revascularization between patients with and without baseline depressive symptoms. Thus, the presence of depressive symptoms may mitigate symptomatic relief associated with revascularization. Similarly, when stratified by baseline SAQ scores using the median as a cutoff, subjects with worse (<median) angina, physical limitation and disease perception had significant improvement in depressive symptoms post-revascularization compared to those with SAQ scores zmedian (Fig. 4B).

\section{Discussion}

In this large study of patients undergoing left heart catheterization for evaluation of $\mathrm{CAD}$, we found that depressive symptoms are robustly associated with self-reported increased frequency of chest pain, physical limitation, and disease perception. This finding is of particular interest in subjects with minimal and non-obstructive coronary atherosclerosis on angiogram in whom depressive symptoms were also significantly associated with chest pain and physical limitation. The association persisted at follow-up, providing validation of our 
findings at baseline. Depressive symptoms were the most important predictor of chest pain, contributing to at least $70 \%$ of the variance in models predicting chest pain that incorporate traditional risk factors as well as coronary artery disease severity. Additionally, patients who reported improvement in depressive symptoms during follow-up also noted decreased angina frequency, physical limitations and improved disease perception, regardless of whether they had undergone revascularization or were on antidepressants. Finally, in exploratory analyses we observed that patients without depressive symptoms had greater symptom relief after revascularization compared to those with depressive symptoms.

Previous studies have also reported a similar relationship between depression and angina. For example, veterans with stable $\mathrm{CAD}$ and a new diagnosis of depression at follow-up reported more angina, physical limitation and lower treatment satisfaction [24]. Amore recent study in patients with non-obstructive CAD identified an association between type $\mathrm{D}$ personality - characterized by negative affective states - and increased frequency of chest pain [13].We have previously reported that depression in women after myocardial infarction was associated worse angina and physical limitation in addition to worse outcomes [25]. The present study further characterizes the association between depressive symptoms and chest pain using validated questionnaires in a large cohort of both men and women with angiographic data and detailed clinical characteristics, and shows that this relationship persists irrespective of the presence and severity of obstructive CAD.

Although cause and effect relationship cannot be established from this observational study, our findings support the existence of a bidirectional relationship between depressive symptoms and chest pain in patients with CAD. On the one hand, depressive symptoms improved after revascularization, suggesting that chest pain may be driving depression. On the other hand, we show that chest pain was worse in patients with depressive symptoms regardless of the presence of $\mathrm{CAD}$, and that revascularization produced more effective relief from chest pain in those without depressive symptoms. Thus, depressive symptoms may worsen perception of chest pain and culminate in a worse quality of life. In a longitudinal, population based study, baseline depression was associated with incident pain [26]. Similarly, persons with chronic pain often develop depressive symptoms [27]. While a direct causal link is yet to be established, depression is hypothesized to affect cardiovascular health via several pathophysiologic mechanisms including increased exposure to risk factors and high risk behaviors, heightened sympathetic drive, neuro-hormonal disturbances, vascular dysfunction and chronic inflammation [28,29]. Depression and chest pain may thus be related through neurologic mechanisms or endothelial function, rather than by worsening of epicardial CAD [30].

Several meta-analyses have reviewed randomized trials designed to treat chest pain in patients with non-obstructive CAD using pharmacologic and non-pharmacologic antidepressant therapies. Although cognitive behavioral therapy appears to have at least a modest benefit in decreasing chest pain frequency [31], studies examining antidepressant therapies have been inconclusive [31-33].Most trials examining the use of antidepressants for the relief of non-cardiac chest pain have had small sample sizes and have excluded patients with known diagnoses of depression and CAD [31-33]. The results of these trials may also have been confounded by ineffective antidepressant therapy, as follow-up 
depressive symptoms have either not been reported or did not show a sustained decrease. In addition, antidepressant therapy may be effective in only a subset of patients with chest pain. In our study, the association between depressive symptoms and angina was dampened in patients receiving treatment for depression, suggesting that antidepressant therapy may mitigate the psychological component contributing to chest pain.

Limitations of our study include the observational nature of the study that precludes definitive conclusions regarding causation. The post-revascularization findings are also in a smaller group completing the follow-up questionnaires, and thus are exploratory and hypothesis generating given the sample is likely biases towards survivors at 6-24 months. Strengths include the large sample size, diversity of the cohort, the detailed characterization, and use of validated PHQ-8 and SAQ questionnaires that have been associated with outcomes $[34,35]$. Moreover, replication of baseline findings at follow-up lends further validity to our findings.

\title{
4.1. Implications
}

Despite abundant evidence regarding the impact of depression on CAD and its outcomes, it is yet to be incorporated in the evaluation of the patient with chest pain [36,37]. This is at least partly due to the incomplete understanding of how depression is associated with chest pain and CAD, and the lack of available evidence-based strategies to abrogate that risk. Our findings confirm the existence of a strong association between depressive symptoms and chest pain that is independent of CAD severity, and points to the bidirectional nature of that relationship. Most importantly, it highlights depression as a potential therapeutic target in the treatment of chest pain. Definitive trials are needed to determine whether treatment of depression contributes to the relief of angina in subjects with and without CAD.

\section{Supplementary Material}

Refer to Web version on PubMed Central for supplementary material.

\section{Acknowledgments}

\author{
Funding sources \\ AAQ is supported by 5P01HL101398-02, 1P20HL113451-01, 1R56HL126558-01, 1RF1AG051633-01, R01 \\ NS064162-01, R01 HL89650-01, HL095479-01, 1U10HL110302-01, 1DP3DK094346-01, 2P01HL086773-06A1. \\ SSH is supported by the Abraham J. \& Phyllis Katz Foundation (Atlanta, GA).
}

We would like to thank all members of the Emory ACTSI for their support in performing this study.

\section{References}

1. Mozaffarian D, Benjamin EJ, Go AS, Arnett DK, Blaha MJ, Cushman M, et al. Heart disease and stroke statistics-2015 update: a report from the American Heart Association. Circulation. 2015; 131(4):e29-322. [PubMed: 25520374]

2. Lichtman JH, Froelicher ES, Blumenthal JA, Carney RM, Doering LV, Frasure-Smith N, et al. Depression as a risk factor for poor prognosis among patients with acute coronary syndrome: systematic review and recommendations: a scientific statement from the American Heart Association. Circulation. 2014; 129(12):1350-1369. [PubMed: 24566200] 
3. Robson J, Ayerbe L, Mathur R, Addo J, Wragg A. Clinical value of chest pain presentation and prodromes on the assessment of cardiovascular disease: a cohort study. BMJ Open. 2015; 5(4):e007251.

4. Swap CJ, Nagurney JT. Value and limitations of chest pain history in the evaluation of patients with suspected acute coronary syndromes. JAMA. 2005; 294(20):2623-2629. [PubMed: 16304077]

5. McConaghy JR, Oza RS. Outpatient diagnosis of acute chest pain in adults. Am. Fam. Physician. 2013; 87(3):177-182. [PubMed: 23418761]

6. Loerbroks A, Bosch JA, Mommersteeg PM, Herr RM, Angerer P, Li J. The association of depression and angina pectoris across 47 countries: findings from the 2002 World Health Survey. Eur. J. Epidemiol. 2014; 29(7):507-515. [PubMed: 24952719]

7. Wheeler A, Schrader G, Tucker G, Adams R, Tavella R, Beltrame JF. Prevalence of depression in patients with chest pain and non-obstructive coronary artery disease. Am. J. Cardiol. 2013; 112(5): 656-659. [PubMed: 23711812]

8. Huffman JC, Celano CM, Beach SR, Motiwala SR, Januzzi JL. Depression and cardiac disease: epidemiology, mechanisms, and diagnosis. Cardiovasc. Psychiatry Neurol. 2013; 2013:695925. [PubMed: 23653854]

9. Kessler RC, Berglund P, Demler O, Jin R, Koretz D, Merikangas KR, et al. The epidemiology of major depressive disorder: results from the National Comorbidity Survey Replication (NCS-R). JAMA. 2003; 289(23):3095-3105. [PubMed: 12813115]

10. Perk J, De Backer G, Gohlke H, Graham I, Reiner Z, Verschuren M, et al. European guidelines on cardiovascular disease prevention in clinical practice (version 2012). The fifth joint task force of the European Society of Cardiology and Other Societies on cardiovascular disease prevention in clinical practice (constituted by representatives of nine societies and by invited experts). Eur. Heart J. 2012; 33(13):1635-1701. [PubMed: 22555213]

11. Shah AJ, Ghasemzadeh N, Zaragoza-Macias E, Patel R, Eapen DJ, Neeland IJ, et al. Sex and age differences in the association of depression with obstructive coronary artery disease and adverse cardiovascular events. J. Am. Heart Assoc. 2014; 3(3):e000741. [PubMed: 24943475]

12. Fasick V, Spengler RN, Samankan S, Nader ND, Ignatowski TA. The hippocampus and TNF: common links between chronic pain and depression. Neurosci. Biobehav. Rev. 2015; 53:139-159. [PubMed: 25857253]

13. Mommersteeg PM, Widdershoven JW, Aarnoudse W, Denollet J. Personality subtypes and chest pain in patients with nonobstructive coronary artery disease from the TweeSteden mild stenosis study: mediating effect of anxiety and depression. Eur. J. Pain. 2016; 20(3):427-437. [PubMed: 26105088]

14. Eapen DJ, Manocha P, Ghasemzadeh N, Patel RS, Al Kassem H, Hammadah M, et al. Soluble urokinase plasminogen activator receptor level is an independent predictor of the presence and severity of coronary artery disease and of future adverse events. J. Am. Heart Assoc. 2014; 3(5):e001118. [PubMed: 25341887]

15. Neeland IJ, Patel RS, Eshtehardi P, Dhawan S, MC MD, Rab ST, et al. Coronary angiographic scoring systems: an evaluation of their equivalence and validity. Am. Heart J. 2012; 164(4):547-52 e1. [PubMed: 23067913]

16. Gensini GG. A more meaningful scoring system for determining the severity of coronary heart disease. Am. J. Cardiol. 1983; 51(3):606. [PubMed: 6823874]

17. Kroenke K, Spitzer RL, Williams JB. The PHQ-9: validity of a brief depression severity measure. J. Gen. Intern. Med. 2001; 16(9):606-613. [PubMed: 11556941]

18. Spitzer RL, Kroenke K, Williams JB. Validation and utility of a self-report version of PRIME-MD: the PHQ primary care study. Primary care evaluation of mental disorders. Patient health questionnaire. JAMA. 1999; 282(18):1737-1744. [PubMed: 10568646]

19. Lowe B, Unutzer J, Callahan CM, Perkins AJ, Kroenke K. Monitoring depression treatment outcomes with the patient health questionnaire-9. Med. Care. 2004; 42(12):1194-1201. [PubMed: 15550799]

20. Spertus JA, Winder JA, Dewhurst TA, Deyo RA, Prodzinski J, McDonell M, et al. Development and evaluation of the Seattle Angina Questionnaire: a new functional status measure for coronary artery disease. J. Am. Coll. Cardiol. 1995; 25(2):333-341. [PubMed: 7829785] 
21. Stewart A. Conceptual and methodologic issues in defining quality of life: state of the art. Prog. Cardiovasc. Nurs. 1992; 7(1):3-11.

22. Tonidandel S, LeBreton JM. Relative importance analysis: a useful supplement to regression analysis. J. Bus. Psychol. 2011; 26(1):1-9.

23. Akaah IP, Korgaonkar PK. A conjoint investigation of the relative importance of risk relievers in direct marketing. J. Advert. Res. 1988; 28(4):38-44.

24. Trivedi R, Gerrity M, Rumsfeld JS, Spertus JA, Sun H, McDonell M, et al. Angina symptom burden associated with depression status among veterans with ischemic heart disease. Ann. Behav. Med. 2015; 49(1):58-65. [PubMed: 25008432]

25. Parashar S, Rumsfeld JS, Spertus JA, Reid KJ, Wenger NK, Krumholz HM, et al. Time course of depression and outcome of myocardial infarction. Arch. Intern. Med. 2006; 166(18):2035-2043. [PubMed: 17030839]

26. Calvó-Perxas L, Vilalta-Franch J, Turró-Garriga O, López-Pousa S, Garre-Olmo J. Gender differences in depression and pain: a two year follow-up study of the survey of health, ageing and retirement in Europe. J. Affect. Disord. 2016; 193:157-164. [PubMed: 26773909]

27. Linton SJ, Bergbom S. Understanding the link between depression and pain. Scand. J. Pain. 2011; 2(2):47-54.

28. Brotman DJ, Golden SH, Wittstein IS. The cardiovascular toll of stress. Lancet. 2007; 370(9592): 1089-1100. [PubMed: 17822755]

29. Mensah GA, Mokdad AH, Ford ES, Greenlund KJ, Croft JB. State of disparities in cardiovascular health in the United States. Circulation. 2005; 111(10):1233-1241. [PubMed: 15769763]

30. Vaccarino V, Votaw J, Faber T, Veledar E, Murrah NV, Jones LR, et al. Major depression and coronary flow reserve detected by positron emission tomography. Arch. Intern. Med. 2009; 169(18):1668-1676. [PubMed: 19822823]

31. Kisely SR, Campbell LA, Yelland MJ, Paydar A. Psychological interventions for symptomatic management of non-specific chest pain in patients with normal coronary anatomy. Cochrane Database Syst. Rev. 2015; 6(6):CD004101.

32. Atluri DK, Chandar AK, Fass R, Falck-Ytter Y. Systematic review with meta-analysis: selective serotonin reuptake inhibitors for noncardiac chest pain. Aliment. Pharmacol. Ther. 2015; 41(2): 167-176. [PubMed: 25412947]

33. Nguyen TM, Eslick GD. Systematic review: the treatment of noncardiac chest pain with antidepressants. Aliment. Pharmacol. Ther. 2012; 35(5):493-500. [PubMed: 22239853]

34. Pressler SJ, Subramanian U, Perkins SM, Gradus-Pizlo I, Kareken D, Kim J, et al. Measuring depressive symptoms in heart failure: validity and reliability of the patient health questionnaire-8. Am. J. Crit. Care. 2011; 20(2):146-152. [PubMed: 20378777]

35. Kimble LP, Dunbar SB, Weintraub WS, McGuire DB, Fazio S, De AK, et al. The Seattle angina questionnaire: reliability and validity in women with chronic stable angina. Heart Dis. 2002; 4(4): 206-211. [PubMed: 12147179]

36. Steptoe A, Kivimaki M. Stress and cardiovascular disease. Nat. Rev. Cardiol. 2012; 9(6):360-370. [PubMed: 22473079]

37. Schnohr P, Marott JL, Kristensen TS, Gyntelberg F, Gronbaek M, Lange P, et al. Ranking of psychosocial and traditional risk factors by importance for coronary heart disease: the Copenhagen City Heart Study. Eur. Heart J. 2015; 36(22):1385-1393. [PubMed: 25681607] 
A

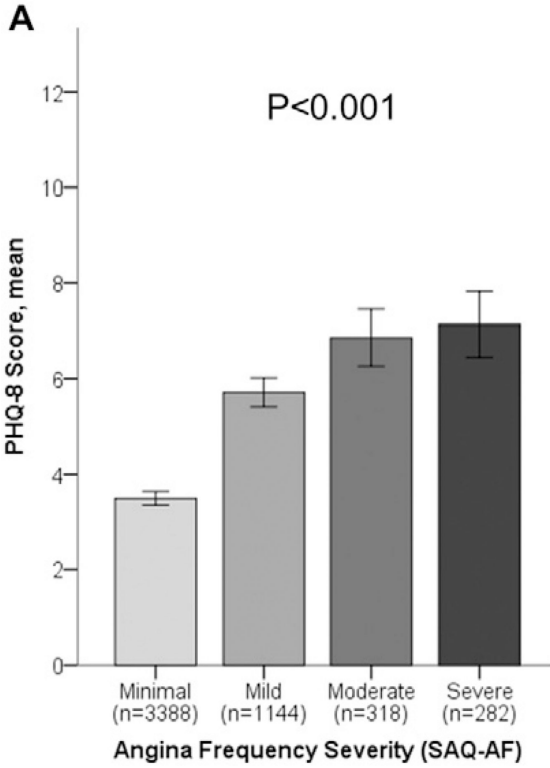

B

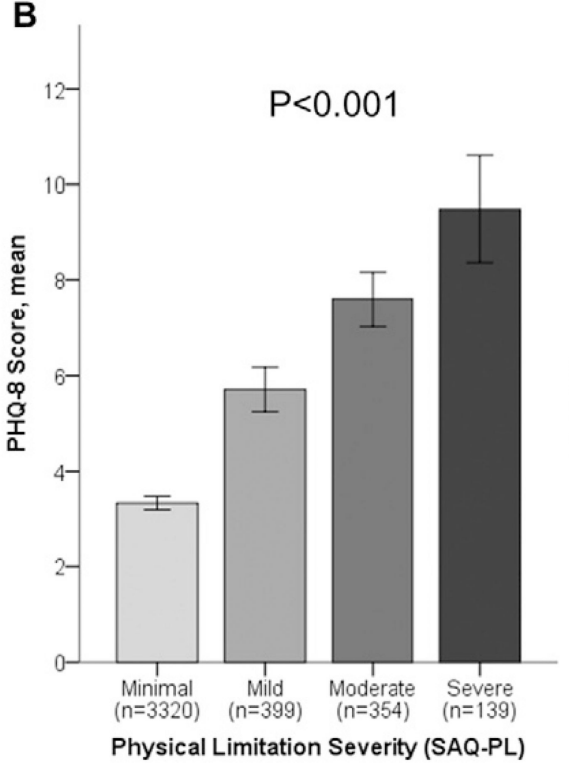

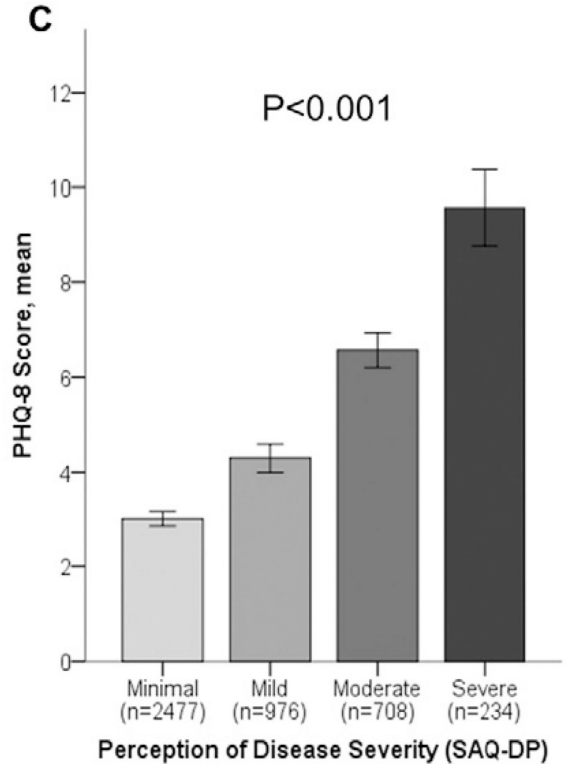

Fig. 1.

PHQ-8 scores stratified by subscales of the Seattle Angina Questionnaire. Panels A, B, and C depict the mean PHQ-8 score stratified by SAQ subscale severity; angina frequency (SAQ-AF), physical limitation (SAQ-PL) and disease perception (SAQ-DP) respectively. Severity cut-offs for all three scores are 75-100 for minimal, 50-74 for mild, 25-49 for moderate, and 0-24 for severe. Error bars represent 95\% confidence interval. $P$-Value was determined using the non-parametric Kruskal-Wallis test. 
A Angina Frequency (SAQ-AF)

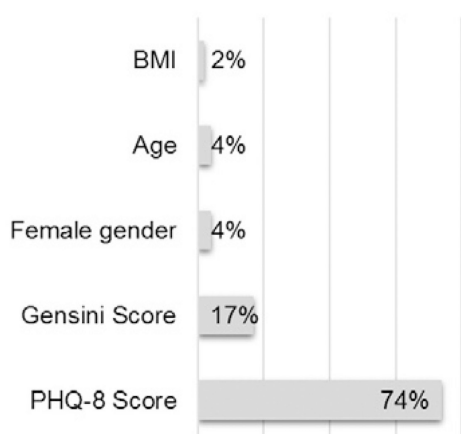

$\begin{array}{llllll}0 \% & 20 \% & 40 \% & 60 \% & 80 \% & 100 \%\end{array}$
B Physical Limitations (SAQ-DS)

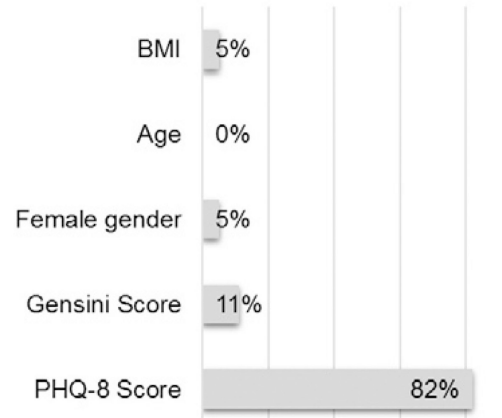

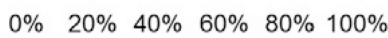

C Perception of Disease Severity

(SAQ-DS)

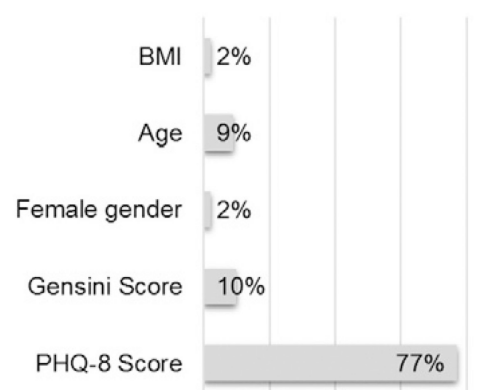

$\begin{array}{llllll}0 \% & 20 \% & 40 \% & 60 \% & 80 \% & 100 \%\end{array}$

Fig. 2.

Significant predictors of SAQ subscales at baseline ranked by importance. Variables displayed are the statistically significant predictors of SAQ subscales. Importance denotes the percentage of variance in the model that the independent variable contributes to. Gensini score quantifies coronary artery disease severity on a continuous scale. 

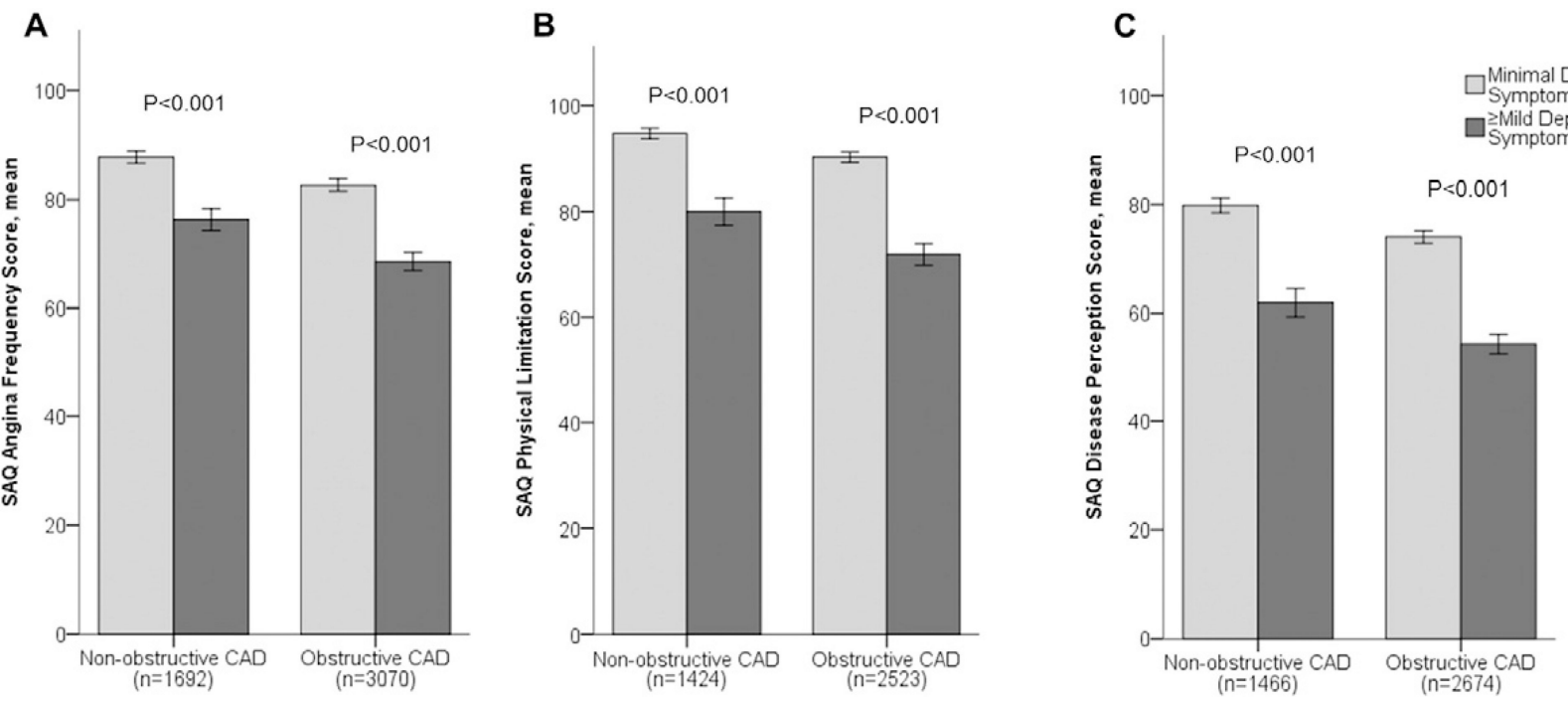

Fig. 3.

SAQ subscales stratified by coronary artery disease severity and depression. Panels A, B and $\mathrm{C}$ depict the mean SAQ subscale score; angina frequency (SAQ-AF), physical limitation (SAQ-PL) and disease perception (SAQ-DP), respectively, stratified by presence or absence of obstructive coronary artery disease (CAD) and depressive symptoms. Obstructive CAD is defined as the presence of a $250 \%$ obstructive lesion in any of the major vessels on coronary angiogram at enrollment. Displayed $P$-values are for the comparisons between PHQ-8 $<5$ and PHQ-8 $\geq 5$ in each stratum. Error bars represent 95\% confidence interval. 
A Baseline-adjusted Change in SAQ-Scores at Follow-up After Revascularization

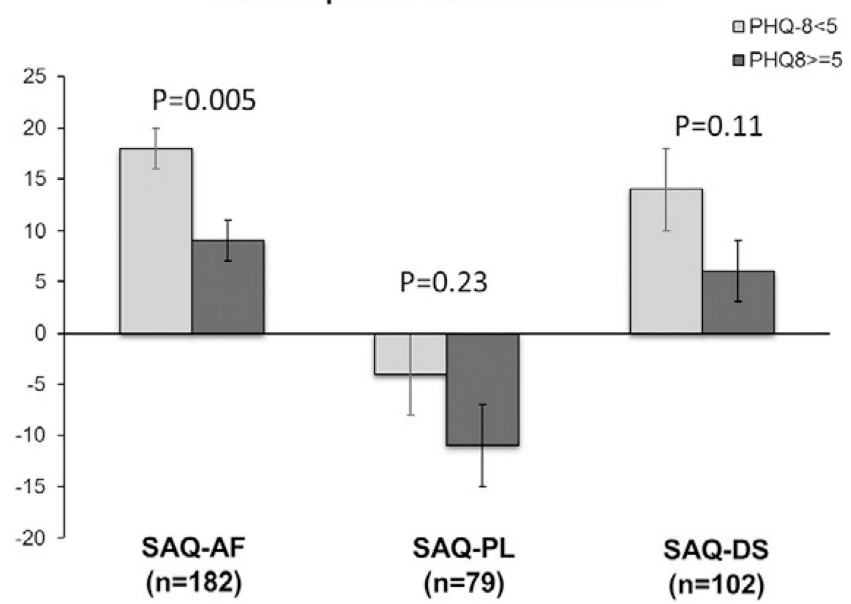

B Baseline-adjusted Change in PHQ-8 Scores at Follow-up After Revascularization

$\square S A Q<$ median $\square \mathrm{SAQ}>=$ median

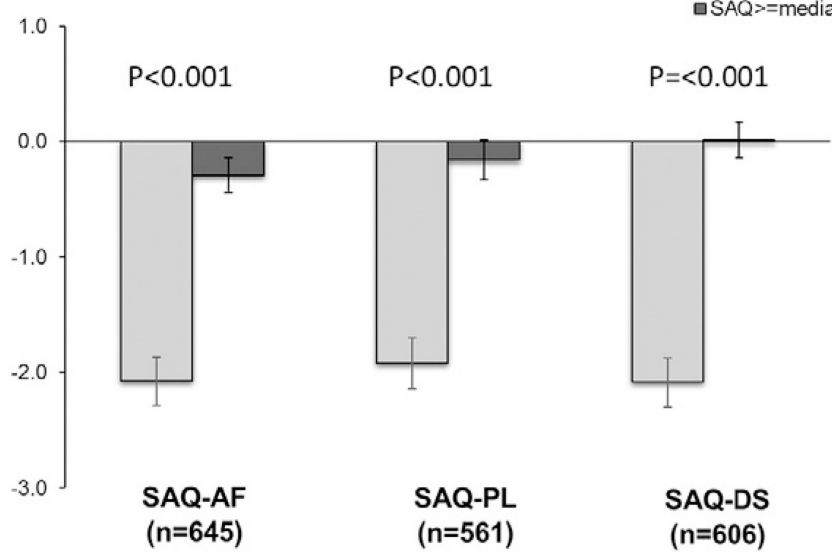

Fig. 4.

Baseline-adjusted change in SAQ subscales at follow-up after revascularization in patients with and without depressive symptoms at baseline. Panel A depicts the baseline-adjusted change in SAQ subscale score; angina frequency (SAQ-AF), physical limitation (SAQ-PL) and disease perception (SAQ-DP) respectively at post-revascularization follow-up, and stratified by the baseline depressive symptoms (PHQ- $8<$ or 25 ). Panel B depicts the baseline-adjusted change in PHQ-8 score post-revascularization stratified by baseline median SAQ-score. A cutoff of 80was used for SAQ-AF, 100 for SAQ-PL and 67 for SAQDS. Data shown are from subsets of patients with available enrollment and postrevascularization PHQ-8 and SAQ scores. Error bars represent \pm 1 standard error. 
Table 1

Demographics and clinical characteristics of the study population.

\begin{tabular}{|c|c|c|c|c|}
\hline Variables & $\begin{array}{l}\text { Entire Cohort } \\
(n=5158)\end{array}$ & $\begin{array}{l}\text { Minimal Depressive } \\
\text { Symptoms }(\text { PHQ-8 < 5) } \\
(n=3256)\end{array}$ & $\begin{array}{l}\geq \text { Mild Depressive Symptoms } \\
(\text { PHQ-8 } \geq 5) \\
(n=1902)\end{array}$ & $P$-Value \\
\hline Age, years & $63(12)$ & $64(12)$ & $62(13)$ & $<0.001$ \\
\hline Male, n (\%) & $3350(65 \%)$ & $2265(70 \%)$ & $1085(57 \%)$ & $<0.001$ \\
\hline African American, n (\%) & $1042(20 \%)$ & $648(20 \%)$ & $394(21 \%)$ & 0.50 \\
\hline Body Mass Index, $\mathrm{kg} / \mathrm{m}^{2}$ & $30(6)$ & $29(6)$ & $30(7)$ & $<0.001$ \\
\hline \multicolumn{5}{|l|}{ Clinical Characteristics } \\
\hline History of Smoking, n (\%) & $3657(72 \%)$ & $2267(70 \%)$ & $1390(74 \%)$ & 0.016 \\
\hline Hypertension, n (\%) & $3974(78 \%)$ & $2458(77 \%)$ & $1516(81 \%)$ & 0.001 \\
\hline Diabetes Mellitus, n (\%) & $1738(34 \%)$ & $1025(32 \%)$ & $713(38 \%)$ & $<0.001$ \\
\hline Hyperlipidemia, n (\%) & $3582(69 \%)$ & $2222(70 \%)$ & $1360(73 \%)$ & 0.020 \\
\hline Low-Density Lipoprotein, mg/dL & $95(37)$ & $95(37)$ & $94(37)$ & 0.56 \\
\hline High-Density Lipoprotein, mg/dL & $43(14)$ & $43(14)$ & $43(14)$ & 0.23 \\
\hline History of myocardial infarction, $\mathrm{n}(\%)$ & $1220(22 \%)$ & $736(23 \%)$ & $484(26 \%)$ & 0.022 \\
\hline Obstructive coronary artery disease ${ }^{a}, \mathrm{n}(\%)$ & $3083(64 \%)$ & $1933(64 \%)$ & $1150(66 \%)$ & 0.15 \\
\hline Gensini Score, median (IQR) & $11(0,47)$ & $10(0,44)$ & $10(0,45)$ & 0.75 \\
\hline PHQ-8 Score, median (IQR) & $3(0,6)$ & $1(0,3)$ & $8(6,12)$ & $<0.001$ \\
\hline Receiving Therapy for Depression ${ }^{b}, \mathrm{n}(\%)$ & $821(17 \%)$ & $298(10 \%)$ & $523(29 \%)$ & $<0.001$ \\
\hline SAQ Angina Frequency Score < 75, n $(\%)$ & $1744(34 \%)$ & $821(25 \%)$ & $923(49 \%)$ & $<0.001$ \\
\hline SAQ Physical Limitation Score < 75, $\mathrm{n}(\%)$ & $892(21 \%)$ & $342(12 \%)$ & $550(38 \%)$ & $<0.001$ \\
\hline SAQ Disease Perception Score < 75, n (\%) & $1918(44 \%)$ & $986(35 \%)$ & $932(60 \%)$ & $<0.001$ \\
\hline \multicolumn{5}{|c|}{ *Values are mean $(\mathrm{SD}), \mathrm{n}(\%)$ or median $(25$ th, 75 th percentile). as noted. } \\
\hline \multicolumn{5}{|c|}{ Statistically significant $P$-values for the comparison between subjects with minimal and əmild depressive symptoms are bolded. } \\
\hline \multicolumn{5}{|c|}{$\begin{array}{l}\text { abstructive coronary artery disease is defined as the presence of a } \geq 50 \% \text { obstructive lesion in any of the major vessels on coronary angiogram at } \\
\text { enrollment. }\end{array}$} \\
\hline
\end{tabular}

Int J Cardiol. Author manuscript; available in PMC 2018 April 03. 


\section{Table 2}

Multivariable analysis of predictors of SAQ subscales at baseline

\begin{tabular}{|c|c|c|c|}
\hline \multirow[t]{2}{*}{ Variables } & \multicolumn{3}{|l|}{$\beta, P$-value } \\
\hline & SAQ-AF & SAQ-PL & SAQ-DS \\
\hline \multicolumn{4}{|l|}{ Model 1: Clinical Characteristics } \\
\hline Age, per 10 years & $2.28,<0.001$ & $1.22,0.001$ & $4.25,<0.001$ \\
\hline Male & $6.05,<0.001$ & $6.71,<0.001$ & $7.84,<0.001$ \\
\hline African American & $1.96,0.047$ & $1.73,0.11$ & $1.83,0.11$ \\
\hline Body Mass Index, per kg/m² & $-0.20,0.002$ & $-0.39,<0.001$ & $-0.32,<0.001$ \\
\hline History of Smoking & $-0.17,0.84$ & $-0.254,0.78$ & $-1.000,0.30$ \\
\hline Hypertension & $-1.46,0.13$ & $-2.52,0.013$ & $-1.83,0.08$ \\
\hline Diabetes Mellitus & $-0.50,0.55$ & $-0.46,0.61$ & $0.32,0.73$ \\
\hline Hyperlipidemia & $-3.63,<0.001$ & $-2.46, \mathbf{0 . 0 1 0}$ & $-3.33,<0.001$ \\
\hline History of myocardial infarction & $-2.99,0.001$ & $-1.65,0.11$ & $-2.66,0.013$ \\
\hline a) Obstructive Coronary Artery Disease & $-8.97,<0.001$ & $-8.13,<0.001$ & $-11.48,<0.001$ \\
\hline b) Gensini Score, per unit increase & $-0.05,<0.001$ & $-0.05,<0.001$ & $-0.06,<0.001$ \\
\hline \multicolumn{4}{|l|}{ Model 2: Clinical Characteristics and Depressive Symptoms } \\
\hline PHQ-8 score, per unit increase & $-1.30,<0.001$ & $-1.72,<0.001$ & $-2.01,<0.001$ \\
\hline Receiving therapy for depression & $-0.59,0.61$ & $0.67,0.55$ & $1.35,0.25$ \\
\hline \multicolumn{4}{|l|}{ Sensitivity Analyses } \\
\hline PHQ-8 and Gender & $P=0.88$ & $P=0.23$ & $P=0.31$ \\
\hline PHQ-8 and Obstructive Coronary Artery Disease & $P=0.08$ & $P=0.008$ & $P=0.88$ \\
\hline Patients with Obstructive Coronary Artery Disease & $-1.39,<0.001$ & $-1.87,0.008$ & - \\
\hline Patients without Obstructive Coronary Artery Disease & $-1.11,<0.001$ & $-1.40,0.008$ & - \\
\hline PHQ-8 and Therapy for Depression & $P=0.002$ & $P=0.018$ & $P<0.001$ \\
\hline Patients on Therapy for Depression & $-0.88,<0.001$ & $-1.39,<0.001$ & $-1.51,<0.001$ \\
\hline Patients not on Therapy for Depression & $-1.45,<0.001$ & $-1.84,<0.001$ & $-2.22,<0.001$ \\
\hline
\end{tabular}

In Model 1, obstructive coronary artery disease and Gensini score were entered separately in the model. Model 2 included clinical characteristics of Model 1 in addition to the depression-related variables. Sensitivity analyses explored the interactions between PHQ- 8 score and gender, obstructive coronary artery disease, and therapy for depression. Estimates for relevant subgroups were shown for interaction $P$-values $<0.1$. Statistically significant P-values are bolded. 


\section{을 \\ 졸}

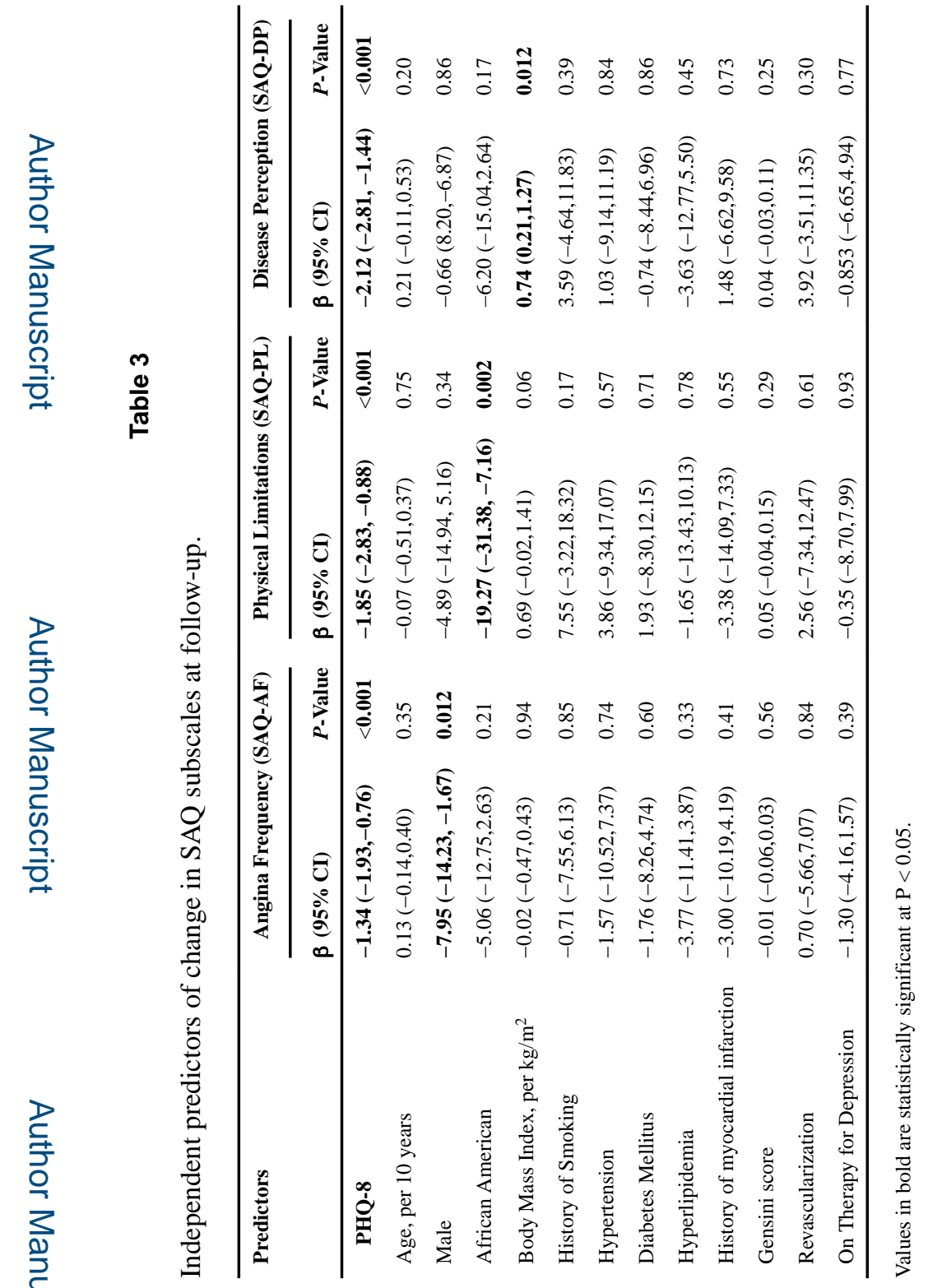

Int J Cardiol. Author manuscript; available in PMC 2018 April 03. 